\title{
Editorial: advances in multimedia and ubiquitous technologies for the new era
}

\author{
Sang-Soo Yeo • Geyong Min • Weifeng Chen
}

Published online: 12 May 2012

(C) Springer Science+Business Media, LLC 2012

\section{Summary}

Recent advances in pervasive computers, networks, telecommunication, and information technology, along with the proliferation of multimedia-capable mobile devices, such as laptops, portable media players, personal digital assistants, and cellular telephones, have stimulated the development of intelligent and pervasive multimedia applications in a ubiquitous environment. The new multimedia standards (for example, MPEG-21) facilitate the seamless integration of multiple modalities into interoperable multimedia frameworks, transforming the way people work and interact with multimedia data. These key technologies and multimedia solutions interact and collaborate with each other in increasingly effective ways, contributing to the multimedia revolution and having a significant impact across a wide spectrum of consumer, business, healthcare, education, and governmental domains. This conference provides an opportunity for academic and industry professionals to discuss recent progress in the area of multimedia and ubiquitous environment including models and systems, new directions, novel applications associated with the utilization and acceptance of ubiquitous computing devices and systems.

We have received 41 manuscripts. After the pre-review process, 28 manuscripts were selected for the first review. Sixteen manuscripts were finally selected for this special

\footnotetext{
S.-S. Yeo $(\bowtie)$

Division of Computer Engineering, Mokwon University, Daejeon 302-729, Korea e-mail: ssyeo2010@gmail.com
}

G. Min

School of Informatics, University of Bradford, Bradford, United Kingdom e-mail: g.min@brad.ac.uk

W. Chen

Department of Mathematics, Computer Science and Information Systems, California University of Pennsylvania, California, PA, USA e-mail: chen@calu.edu 
issue after the first, second review processes. Each manuscript selected was blindly reviewed by at least three reviewers consisting of guest editors and external reviewers.

The first paper entitled "Semantic Media Decision Taking using N3Logic," by Davy Van Deurse et al. proposes a Media Decision Taking Engine (MDTE), enabling the automatic selection and/or rating of multimedia content versions, based on the available context information. The decision rules are based on a rating function, supporting the specification of weights and affinity parameters for each environment property.

The second paper entitled "Context and Profile based Cascade Classifier for Efficient People Detection and Safety Care system," by Miyoung Nam et al. presents a system of extracting and tracking objects for multimedia system, and addresses how to extract the head feature from an object area that is extracted from video via differential imaging from the background image. They discuss how to extract multiple objects and tracking based on context, rather than a single object, via cascade extraction to track more than one human at a time.

Another paper in this special issue "Extraction of Enhanced Evoked Potentials using Wavelet Filtering," by Lee et al. proposes a filtering method using the multi-resolution wavelet transform in order to remove physiological artifacts and gain the improved evoked potentials.

The fourth paper entitled "An Enhanced Motion Estimation Approach Using a Genetic Trail Bounded Approximation for H.264/AVC Codecs," by Kim et al. proposes a robust motion estimation scheme that uses a noble genetic trail bounded approximation (GTBA) approach to speed up the encoding process of H.264/AVC video compression and to reduce the number of bits required to code frame. The proposed algorithm is utilized to enhance the fitness function strength by integrating trail information of motion vector and sum of absolute difference (SAD) information into a fitness function.

The fifth paper entitled "An Analysis of Content-Based Classification of Audio Signals using a Fuzzy C-Means Algorithm," by Kim et al. proposes an efficient content-based audio classification approach to classify audio signals into broad genres using a fuzzy c-means (FCM) algorithm. They analyze different characteristic features of audio signals in time, frequency, and coefficient domains and select the optimal feature vector by employing a noble analytical scoring method to each feature

Next paper entitled "Intelligent Noise Detection and Filtering using Neuro-Fuzzy System," by Arfan Jaffar et al. presents a neuro-fuzzy based blind image restoration to remove impulse noise from low as well as highly corrupted images. Main components of the proposed technique include noise detection, histogram estimation and noise filtering process. Proposed technique constructs the fuzzy sets using fuzzy number construction algorithm.

The seventh paper entitled "Iterative Random Search: A New Local Minima Resistant Algorithm for Motion Estimation in High Definition Videos," by Marcelo Porto et al. presents a new fast algorithm for motion estimation focusing on high definition videos named Iterative Random Search (IRS). The IRS algorithm randomly chooses candidate blocks from the reference frame, which makes it less susceptible to local minimum falls.

The eighth paper entitled "Enriching User Search Experience by Mining Social Streams with Associative Ripples," by Xiaokang Zhou et al. presents a method to analyze and extract meaningful information in accordance with users' current needs and interests from social streams using two developed algorithms, and go further to 
integrate these organized stream data which are described as associative ripples into the search system, in order to improve the relevance of the results obtained from the search engine and feedback users with a new perspective of the sought issues to guide the further information seeking process, which can benefit both search experience enrichment and search process facilitation.

Another paper in this special issue, entitled "A study on stackable mosaic generation for mobile devices," by Yoon et al. presents a mobile device application for converting photographs into photomosaic images. They propose a method that produces a photomosaic effect using a database that consists of rotatable images and a solution to the performance issue based on a best match search.

The tenth paper entitled "Multiple 3D Object Position Estimation and Tracking using Double Filtering on Multi-core Processor," by Jeong et al. proposes a new algorithm to tracking and estimating pose for multiple $3 \mathrm{D}$ objects that has robustness, real-time processing and fast object registration time. Followings are key features of proposed multiple 3D object tracking algorithm. Double robust filtering feature gives robustness and accuracy. Using parallel implementation on multi-core processor and ROI, real-time processing is possible. And, fast object registration is accomplished by SURF feature extractor.

The eleventh paper entitled "VoIP Encryption Module," by Son et al. proposes the VoIP encryption module for securing privacy. In encoding communication using the proposed module, the AES advised that RFC (Request for Comments) 3711 should be used which resulted in the overall verification of the system performance and delay times through experiments.

Next paper entitled "Towards a cultural user interface generation principles," by Oh et al. proposes 3-D integrated design principles of modeling, design, and system for the design of cultural user interface(UI) generation reflecting the users' potential culture models. The studies with regard to UI generation have been focused on UI pattern models, but in terms of smart UI generation development, considering the cultural environment of users is required.

The thirteenth paper entitled "A Service Component based CAT system with SCORM for Advanced Learning Effects," by Hong et al. implements logic as a service component. The system process performs the learning test program's logic by interface between the service components. The structure of the system interlinks SCORM API with the learning contents and items through a separate e-learning server.

Next paper entitled "An Object-based Middleware Supporting Efficient Interoperability on a Smart Home Network," by Kim et al. demonstrates that association rule mining based on the dependence between appliances is feasible and its performance is very much comparable with that of an existing association rule mining algorithm.

The fifteenth paper entitled "An object expression system using depth-maps," by Lee et al. presents an object expression system that uses depth-maps for interactions without any additional input devices in order to improve immersiveness. By using this system, the use of 3D modelers could be excluded when 3D objects were created; thus, the efficiency of object creation could be improved.

The last paper entitled "Extraction of Ridge and Valley Lines from Unorganized Points," by Kim et al. computes neighbor information using a Delaunay tessellation. Ridge and valley points can then be detected as zero-crossings, and connected using curvature directions. They also demonstrate their method on several large point-sampled models, rendered by point-splatting, on which the ridge and valley lines are rendered with line width determined from curvatures. 


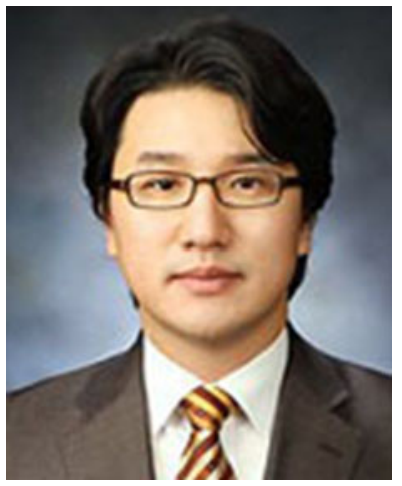

Dr. Sang-Soo Yeo received his master's degree and Ph.D degree in Computer Science \& Engineering from Chung-Ang University, Seoul, Korea. He was a visiting scholar at Kyushu University, Japan. He worked for BTWorks, Inc. as a General Manager, and at the same time was an adjunct professor at Hannam University. He is currently a professor at the Division of Computer Engineering, Mokwon University, Korea. He is Vice President of Future Technology Research Association International (FTRA) and Vice President of Korea Information Technology Convergence Society. He has been serving as various chair positions in a number of conferences and workshops; MUE 2007, IPC-07, FBIT 2007, FGCN 2007, WPS 2008, SH'07, MUE 2008, ISA 2008, CSA 2008, UMC 2008, BSBT 2008, FGCN 2008, ASEA 2008, SecTech 2008, CSA 2009, EM-Com 2009, ICUT 2009, FutureTech 2010, ICA3PP 2010, ITCS 2010, EMC 2010, MUE 2010, HumanCom 2010, ACSA 2010, ISPA 2011, ICASE 2011, MUE 2011, FutureTech 2011, GSDP 2011, ITCS 2011, STA 2011, and PDCAT 2011. Dr. Yeo's research interests include Security, Ubiquitous Computing, Multimedia Service, Ubiquitous Computing, Embedded System, and Bioinformatics. He is a member of the KIPS, KIISE, KICS, KIISC, KMS, KDAS, KONI, KIMICS, KIIT, IEEE, and IEEE CS.

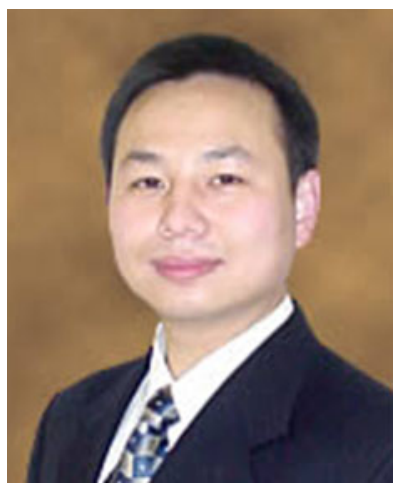

Dr. Geyong Min is currently a senior lecturer in the Department of Computing at the University of Bradford. He was awarded the $\mathrm{PhD}$ degree in Computing Science from the University of Glasgow in the United Kingdom. He conducts research in the general areas of Design and Performance Analysis of Computer Networks and Communication Systems including Internet, Wireless Networks and Mobile Systems, and Interconnection Networks for Parallel and Grid Computing Systems. His research results have been accepted for publication in the wellestablished journals including IEEE Transactions on Computers, IEEE Transactions on Multimedia, IEE Proceedings-Computers and Digital Techniques, Performance Evaluation, Journal of Computer Networks, Journal of Mobile Networking and Applications, Journal of Supercomputing, Journal of Computers \& Electrical Engineering, and in the reputable conferences such as IEEE/ACM Int. Parallel \& Distributed Processing Symposium (IPDPS), IEEE/ACM Int. Symposium on Modeling, Analysis and Simulation of Computer and Telecommunication Systems (MASCOTS), and IEEE Symposium on Computers and Communications (ISCC), etc. Dr. Min is the Founding Co-Chair of the International Workshop on Performance Modelling, Evaluation, and Optimisation of Parallel and Distributed Systems (PMEO-PDS) held in conjunction with IEEE/ACM-IPDPS, and the International 
Workshop on Performance Analysis and Enhancement of Wireless Networks (PAEMN) held in conjunction with IEEE AINA conference. He serves as an Editorial Board member of the Journal of Wireless and Mobile Computing and the Guest Editor for the journals of Computation and Concurrency: Practice and Experience, Future Generation Computer Systems, Supercomputing, and Cluster Computing. He is the Program Vice-Chair of International Conference on High Performance Computing and Communications (HPCC'2005) and International Conference on Wireless Networks (ICWN'2005). He served on program committees of 30 professional conferences/workshops including GLOBECOM and ICCCN. He is a member of the IEEE Computer Society. He is the Co-Editor a new book (Performance Evaluation of Parallel, Distributed and Emergent Systems, Nova Science Publishers, 2005).

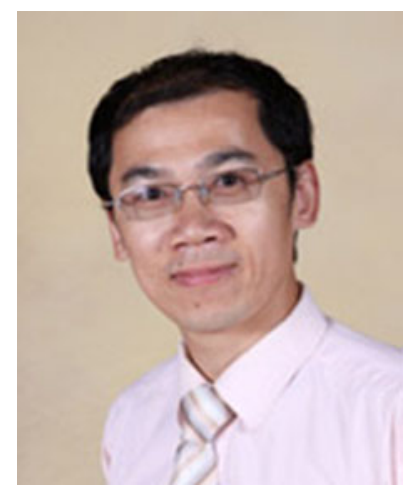

Dr. Weifeng Chen received his Ph.D. degree in Computer Science, University of Massachusetts at Amherst in 2006. He is currently a professor at department of mathematics, computer science and information systems, California University of Pennsylvania, USA. His main research interests include Computer forensic, trust negotiation, privacy protection, key management, intrusion detection, and authentication in security \& privacy area and publish/subscribe systems, optimization, performance evaluation, measurement and traffic analysis in computer networking area. 